\title{
Treg und Th17-Zellen: Verwandtschaft mit Folgen
}

\author{
Plastizität von Th17-Helfer-T-Zellen und regulatorischen T-Zellen (Treg) bei oligo- \\ und polyartikulärer Juveniler Idiopathischer Arthritis im Kindesalter
}

\section{Treg and Th17 cells: kinship with consequences}

\author{
Plasticity of Th17 helper T cells and regulatory T cells (Treg) in oligoarticular and \\ polyarticular Juvenile Idiopathic Arthritis
}

Autorin

Martina Prelog

Institut

Universitäts-Kinderklinik Würzburg

Schlüsselwörter

Th17, Treg, Autoimmunarthritis, Plastizität

Keywords

Th17, Treg, autoimmune arthritis, plasticity

Bibliografie

DOI https://doi.org/10.1055/a-1098-6894

arthritis + rheuma 2020; 40: 118-123

(c) Georg Thieme Verlag KG Stuttgart · New York

ISSN 0176-5167

Korrespondenzadresse

Prof. Dr. med. Martina Prelog, MSc

Universitäts-Kinderklinik Würzburg

Pädiatrische Rheumatologie/Spezielle Immunologie

Josef-Schneider-Str. 2, 97080 Würzburg

Prelog_M@ukw.de

\section{ZUSAMMENFASSUNG}

Verschiedene Helfer-T-Zellen (Th) unterscheiden sich in Merkmalen und Funktionen. Th1 werden durch IL-12/IFN-gamma induziert, exprimieren T-bet und produzieren IFN-gamma. IL-17-produzierende Th17 exprimieren RORC, tragen den Chemokinrezeptor CCR6 und werden durch IL-1beta/IL-6/IL23 stimuliert. Regulatorische T-Zellen (Treg) werden durch IL-2/ TGF-beta stimuliert, exprimieren FoxP3 und produzieren IL-10. Th1 sind physiologischerweise an der intrazellulären Erregerabwehr beteiligt, Th2 von Parasiten, Th17 von extrazellulären

Bakterien und Pilzen und Treg an der peripheren T-ZellHomöostase. Eine Dysbalance zwischen Treg/Th1 und Th17 wird mit Autoimmunprozessen in Zusammenhang gebracht. Insbesondere synoviale Th1/Th17-Zellen, die CD161 exprimieren, zeigen enge Korrelationen mit schweren Verläufen der JIA, während IL-17-produzierende Treg/Th17-Zellen pro- und antiinflammatorische Fähigkeiten vereinen können. Alle Th weisen eine hohe Plastizität in unterschiedlichen inflammatorischen Milieus auf. Es gibt Hinweise, dass eine effiziente Hemmung von bestimmten Zytokinen zu einer Rekonstitution der Treg-Funktion beiträgt, was in neuen Therapieansätzen genutzt werden könnte.

\section{ABSTRACT}

Different Thelper cell types (Th) demonstrate different features and functions. Th1 are stimulated by IL-12/IFN-gamma, express T-bet and produce IFN-gamma. IL-17-producing Th17 express RORC, are positive for chemokine receptor CCR6 and are stimulated by IL-1 beta/IL-6/IL-23. IL-2 and TGF-beta stimulate Treg, which express FoxP3 and produce IL-10. Physiologically, Th1 are directed against intracellular pathogens, whereas Th2 fend off parasites, and Th17 extracellular bacteria and fungi. Treg are involved in the peripheral T cell homeostasis. A dysbalance between Treg and Th 1 as well as Th17 is critical in the pathogenesis of many T cell-mediated autoimmune disorders. Particularly, synovial Th1/Th17 cells expressing CD161 correlate with severe courses of JIA. IL-17-producing Treg/Th17 cells combine proand anti-inflammatory abilities. All Th subtypes demonstrate a high grade of plasticity in different inflammatory cytokine milieus. There is upcoming evidence, that efficient blockade of certain cytokines may contribute to the reconstitution of Treg function, which may be utilized in novel therapeutic approaches.
Die Juvenile Idiopathische Arthritis (JIA) stellt eine heterogene Gruppe von Autoimmunarthritis bei Kindern und Jugendlichen dar, die durch eine Beteiligung des angeborenen und erworbenen (adaptiven) Immunsystems gekennzeichnet ist. Während bei der systemischen Form der JIA (Morbus Still) die Aktivierung von Ma- krophagen und des Inflammasoms im Vordergrund steht, sind bei den oligo- und polyartikulären Formen der JIA die Zellen des erworbenen Immunsystems durch die Aktivierung von proinflammatorischen potenziell autoreaktiven T-Helfer-Zellen (Th) bei der Entzündungsreaktion wesentlich beteiligt [1]. 
- Tab. 1 Die wichtigsten Helfer-T-Zell-Subtypen bei der Inflammation im Überblick.

\begin{tabular}{|c|c|c|c|c|c|c|}
\hline & $\begin{array}{l}\text { Induktion } \\
\text { durch }\end{array}$ & $\begin{array}{l}\text { Transkriptions- } \\
\text { faktor }\end{array}$ & $\begin{array}{l}\text { Typische Chemokin- } \\
\text { rezeptoren und } \\
\text { Oberflächenmarker }\end{array}$ & $\begin{array}{l}\text { Schlüssel- } \\
\text { Zytokine }\end{array}$ & Physiologische Rolle & Pathologische Rolle \\
\hline Th1 & IL-12, IFN-Y & T-bet, STAT4 & CXCR3, CCR5 & IFN-y, IL-2, & intrazelluläre Pathogene & Autoimmunität \\
\hline Th2 & IL-4, IL-2 & GATA-3, STAT5 & CCR3, CCR4 & IL-4, IL-5, IL-13 & extrazelluläre Parasiten & Allergie und Asthma \\
\hline Th17 & $\begin{array}{l}\text { IL-1, IL-6, TGF- } \beta \text {, } \\
\text { IL-21, IL-23 }\end{array}$ & RORC, STAT3 & CCR6 & $\begin{array}{l}\text { IL-17, IL-21, } \\
\text { IL-22 }\end{array}$ & extrazelluläre Bakterien, Pilze & Autoimmunität \\
\hline Treg & TGF- $\beta$, IL-2 & FoxP3, STAT5 & CD25, CCR6 & $\begin{array}{l}\text { TGF- } \beta, \text { IL-35, } \\
\text { IL-10 }\end{array}$ & $\begin{array}{l}\text { Immuntoleranz, Lymphozyten- } \\
\text { Homöostase, Regulation von } \\
\text { Immunantworten }\end{array}$ & $\begin{array}{l}\text { Autoimmunität, } \\
\text { Malignome, Allergien }\end{array}$ \\
\hline $\begin{array}{l}\text { Th1/ } \\
\text { Th17 }\end{array}$ & IL-12, IL-1 & RORC, T-bet & CD161, CXCR3, CCR6 & IFN-y, IL-17 & $?$ & $\begin{array}{l}\text { chronische autoimmune } \\
\text { Gelenksinflammation }\end{array}$ \\
\hline $\begin{array}{l}\text { Treg/ } \\
\text { Th17 }\end{array}$ & IL-1, IL-6 & FoxP3, RORC & CCR6 & IL-17 & $\begin{array}{l}\text { Immunhomöostase mit } \\
\text { Mikrobiom im Darm? }\end{array}$ & $\begin{array}{l}\text { Proinflammation/ } \\
\text { Suppression }\end{array}$ \\
\hline
\end{tabular}

Obwohl die Pathogenese der JIA nur rudimentär verstanden ist, zeigen immunsuppressive und immunmodulatorische Therapiestrategien Wirkung im Sinne einer langandauernden Remission der Erkrankung. Insbesondere neue Therapieansätze zielen auf eine Beeinflussung von Entzündungsmediatoren, wie Zytokine (z. B. Tumor-Necrosis-Factor-alpha, TNF- $\alpha$; Interleukin-1beta, IL-1 $\beta$; IL-6; IL-17), ab, was zu einer Immunmodulation des inflammatorischen Milieus vor allem in der Synovialflüssigkeit der betroffenen Gelenke führt.

Dabei sind die vor knapp 2 Jahrzehnten erstmals beschriebene Gruppe von IL-17-produzierenden T-Zellen vom CD4+-HelferT-Zell-Subtyp (Th17) und die Rolle von regulierenden CD4+-Helfer-T-Zellen, den sogenannten Treg-Zellen, zunächst in den wissenschaftlichen und nun auch in den therapeutischen Fokus gerückt. Zahlreiche neuartige Biologika-Therapien und Janus-Kinase-Inhibitoren haben sich als erfolgreiche Hemmer der Differenzierungswege von Th17-Zellen (v. a. von IL-1 $\beta$, des IL-6- und des IL-23Signalweges) oder deren Zytokin-Produkte, wie IL-17A, herausgestellt, die auch klinisch ihre Wirkung bei Autoimmunarthritis im Kindes- und Erwachsenenalter nachweisen konnten. Obwohl die Differenzierung von proinflammatorischen Th17-Zellen und von regulierenden Treg-Zellen determiniert erscheint, zeigten aktuelle Untersuchungen, dass Th17- und Treg-Zellen eine hohe Plastizität aufweisen. Diese Ergebnisse lassen hoffen, dass unter einer geeigneten immunmodulatorischen Therapie protektive Elemente der Treg-Zellen aktiviert und negative Auswirkungen einer überschießenden oder autoreaktiven Th17- und/oder Th1-Reaktion minimiert werden.

Die folgende Übersichtsarbeit soll die Differenzierung der Th17und Treg-Zellen verständlich machen, die Rationale für Therapieansätze erklären und die hohe Plastizität dieser Helfer-T-Zell-Subtypen aufzeigen.

\section{Helfer-T-Zell-Subtypen in der Synovialflüssigkeit von JIA-Patienten}

Noch vor 2 Jahrzehnten differenzierte man die Helfer-T-Zell-Subtypen in 2 Gruppen, Th1- und Th2-Zellen. Die Charakterisierung der IL-17-produzierenden Th17-Zellen und der Treg-Zellen führte zu einem neuen Verständnis der Helfer-T-Zell-Differenzierung. Allen Zellen liegt im Ursprung eine naive T-Zelle (Th0) zugrunde, die sich erst durch Antigen-Erkennung nach Präsentation durch eine Antigen-präsentierende Zelle (APC) abhängig vom umgebenden Zytokin-Milieu in verschiedene Helfer-T-Zell-Subtypen differenziert. Die Differenzierungsmöglichkeiten, die stimulierenden Zytokine, die Charakterisierung der Helfer-T-Zell-Subtypen durch ihre spezifischen Transkriptionsfaktoren und deren charakteristische Chemokinrezeptoren für Migrationsvorgänge, sowie die von den jeweiligen Helfer-T-Zell-produzierten Zytokine und die physiologische und pathogene Rolle der verschiedenen Th-Subtypen sind in - Tab. 1 dargestellt.

Th1-Zellen werden durch Interferon-gamma (IFN-ү) und IL-12 induziert, produzieren selbst IFN- - , exprimieren die Transkriptionsfaktoren T-bet und STAT4 und sind vor allem bei der Abwehr von intrazellulären Pathogenen, wie Viren, beteiligt. Th1-Zellen werden aber vor allem mit dem Anfangsstadium vieler T-Zell-mediierter Autoimmunerkrankungen in Zusammenhang gebracht. CXCR3 stellt einen klassischen Chemokinrezeptor für die Migration dieser Th1-Zellen in das Inflammationsgebiet dar.

Th2-Zellen werden durch IL-4 und IL-2 stimuliert, produzieren IL-4, IL-5 und IL-13, exprimieren die Transkriptionsfaktoren GATA3 und STAT 5 und sind physiologischerweise mit der Immunreaktion gegen extrazelluläre Parasiten, wie Helminthen, assoziiert. Th2 wird eine bedeutende Rolle in der Pathogenese von Allergien und Asthma zugewiesen.

Th17 produzieren IL-17 und werden durch proinflammatorische Zytokine, wie IL-1, IL-6, IL-21, IL-23 und hohe Dosen von Tumor-Growth-Factor-beta (TGF- $\beta$ ), induziert. Für Th17-Zellen ist der Transkriptionsfaktor RORC spezifisch und eine Aktivierung von STAT3 ist typisch. Physiologischerweise sind Th17-Zellen bei der Abwehr gegen extrazelluläre Bakterien, wie z. B. Staphylokokken, und gegen Pilze, wie z. B. Candida albicans, beteiligt. Im Darm spielen Th17-Zellen eine entscheidende Rolle bei der Interaktion und der immunologischen Kontrolle des Darmmikrobioms, wobei hier Th17-Zellen auch eine protektive Aufgabe zugeschrieben wird. Allerdings findet sich eine pathologische Stimulation von Th17-Zel- 


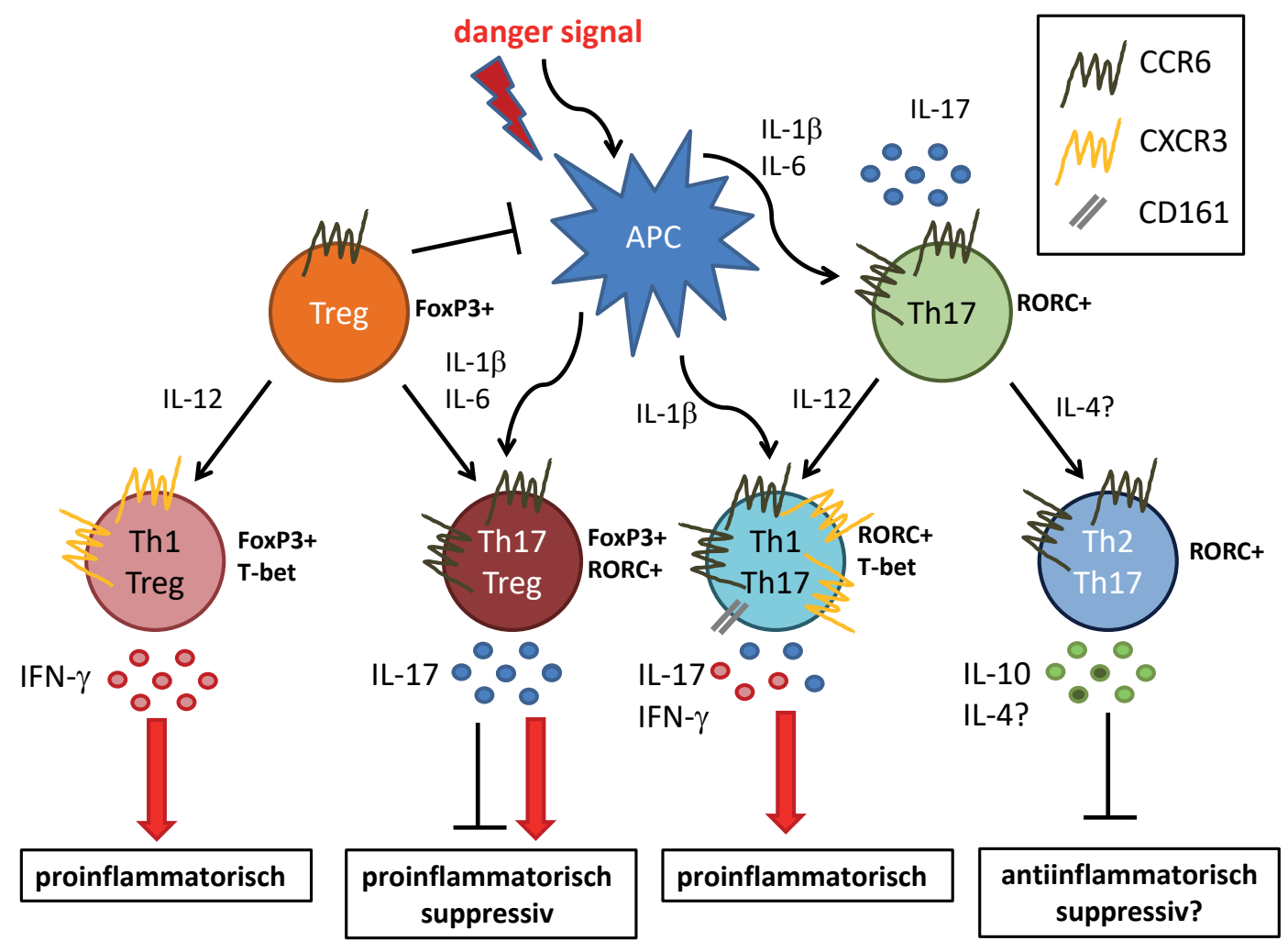

- Abb. 1 Plastizität von humanen Th17- und Treg-Zellen [7, 18].

len bei vielen T-Zell-mediierten Autoimmunerkrankungen. Insbesondere bei der Psoriasis-Arthritis und bei der Enthesitis-assoziierten JIA sowie der Spondyloarthritis wurde IL-17 als wichtiger Entzündungsfaktor identifiziert [2]. Chemokinrezeptor CCR6 ist typischerweise auf Th17-Zellen hochreguliert und vermittelt die Migration in inflammatorisches Gewebe durch Bindung an den Liganden CCL20, der durch inflammatorische Zytokine an Entzündungsorten exprimiert wird.

Regulatorische Helfer-T-Zellen, Treg, werden durch TGF- $\beta$ und IL-2 induziert, exprimieren den Treg-typischen Transkriptionfaktor FoxP3 und STAT5 und produzieren entzündungshemmende Zytokine, wie z. B. IL-10. Treg werden in Thymus-generierte natürliche Treg (nTreg) und in meist an Entzündungsorten entstandenen induzierte Treg (iTreg) unterschieden. Treg vermitteln die periphere Immuntoleranz durch Hemmung von Effektor-Zell-Mechanismen, z. B. durch Aktivierung von CTLA-4, ein hemmendes Molekül der Ko-Stimulation bei Antigen-Präsentation, durch Produktion von IL-10, durch Aktivierung des Fas-FasL-Apoptose-Signalweges und durch Zytotoxizität (z. B. Produktion von Granzyme B) [3]. Treg-Zellen sind somit wesentlich an der peripheren T-Zell-Homöostase, z. B. nach Abklingen eines Infektes oder einer Entzündungsreaktion, beteiligt und regulieren damit Immunantworten. Allerdings werden eine zu starke Reaktion von Treg-Zellen auch mit Malignomentstehung, eine zu schwache Funktion mit der Entstehung von Autoimmunerkrankungen und Allergien in Verbindung gebracht. Insbesondere bei der Autoimmunarthritis zeigt sich eine Treg/Th17-Dysbalance als wichtiger Faktor bei der Pathogenese der Erkrankung [4], wie es auch in der Synovialflüssigkeit von Patienten mit JIA nachweisbar ist [5]. Hierbei zeigt sich auch eine inverse Korrelation zwischen der Expression der Transkriptionsfaktoren RORC und FoxP3 [6].

Als weitere Helfer-T-Zell-Subtypen werden noch sogenannte Th9-Zellen verstanden, die sich durch eine IL-9-Produktion auszeichnen, den Transkriptionsfaktor PU.1 exprimieren und durch TGF- $\beta$ und IL-4 induziert werden. Ob Th9 einen eigenen Helfer-TZell-Subtyp konstituieren oder nur eine weitere Differenzierungsform von inflammatorischen Th2- oder Th17-Zellen darstellen, ist noch unklar. Th9-Zellen werden sowohl mit Autoimmunerkrankungen als auch Allergien in Zusammenhang gebracht und weisen Ähnlichkeiten zu Th2- und Th17-Zellen auf. Th22-Zellen sind durch eine Produktion von IL-22 definiert, weisen aber ansonsten ein ähnliches Profil wie Th17-Zellen auf und werden bei bestimmten Autoimmunerkrankungen, wie z. B. der systemischen Sklerose, vermehrt gefunden. Follikuläre Helfer-T-Zellen werden in Keimzentren gefunden, exprimieren Bcl-6 und sezernieren IL-21. Eine Rolle in der Autoimmunitätsentstehung wird auch diesen Helfer-T-ZellSubtyp zugesprochen.

Bei Kindern mit oligoartikulärer JIA wurde in der Synovia ein weiterer Helfer-T-Zell-Subtyp gefunden, der Eigenschaften von Th1und Th17-Zellen aufweist. Dieser Helfer-T-Zell-Subtyp wird auch als Th1/Th17-Zellen bezeichnet und weist eine Expression von CD161 an der Zelloberfläche auf. CD161 gehört zur Killer cell lectin-like receptor subfamily B der membranständigen Rezeptoren. Es ist jedoch unklar, ob dieses Molekül eine eigene Funktionalität aufweist 
oder nur ein Charakteristikum von Zellen mit erhöhter proinflammatorischer Aktivität (z. B. durch IFN-y- und IL-17-Produktion) oder Zytotoxizität (z. B. auf Natural Killer Zellen und CD8+-zytotoxischen T-Zellen) darstellt. CD161+-Helfer-T-Zellen produzieren typischerweise IFN-y, aber wenig oder kein IL-17, exprimieren jedoch den Th17-typischen Transkriptionsfaktor RORC und IL-23-Rezeptoren (IL-23R). Der IL-23R ist für die Differenzierung von Th17 essenziell, da IL-23 für die Stabilisierung des Th17-Subtyps verantwortlich ist. CD161-exprimierende Th17-Zellen aus der Synovialflüssigkeit von JIA-Patienten konnten in Th1-Zellen verwandelt werden, ein Effekt der durch die Blockade von IL-12 aufgehoben werden konnte [7].

\section{Plastizität von Helfer-T-Zell-Subtypen und Einfluss der Therapie}

Abhängig vom inflammatorischen Milieu können sich naive Th0-Zellen in Th17 oder Treg differenzieren. Gefahrensignale stimulieren APC-Zellen zur Produktion von IL-1 $\beta$ und IL-6, welche verschiedene Th17-Subtypen induzieren, die sowohl regulatorisches Potenzial entfalten können als auch klassisch proinflammatorisch aktiv werden können. Insbesondere unter der Wirkung von niedrigen TGF- $\beta$ und hohen IL-12-Konzentrationen entsteht ein Th1/ Th17-Subtyp, der durch eine hohe Expression von CD161 geprägt ist ( $\triangleright$ Abb. 1). Dieser Subtyp ist vor allem in der Synovialflüssigkeit von JIA-Patienten mit aktiver Arthritis als Marker der Krankheitsaktivität vertreten und korreliert stark mit einer Erhöhung des C-reaktiven Proteins und der Blutsenkungsgeschwindigkeit [8]. Dies unterstreicht die Idee, dass vor allem Th1/Th17-Zellen, die gleichzeitig RORC und T-bet als Transkriptionsfaktoren von Th17 bzw. Th1 exprimieren, im Rahmen der JIA eine entscheidende Rolle bei chronisch inflammatorischen Prozessen spielen könnten [9].

Th17-Zellen zeigen eine hohe Anzahl des TNF-Rezeptors vom Typ II (TNF-RII). Das klassisch proinflammatorische Zytokin TNF- $\alpha$ führt bei der Arthritis zu einem Shift von Th17-Zellen zu Th1/Th17 und zu Th1-Zellen und unterhält damit die Entzündungsreaktion [10]. Damit stammen diese Th1 vom Th17-Subtyp ab und werden auch als „nicht klassische Th1-Zellen“ bezeichnet. Hier spielen auch epigenetische Modifikationen an den Th-typischen Transkriptionsfaktoren eine Rolle. Gerade diese Th1-Zellen, die von den Th17-Zellen abstammen, zeigen eine deutliche Demethylierung am RORC-Transkriptionsfaktor und an den IL-17-codierenden Genen, was zu einer besseren Ablesbarkeit und Expression dieser Gene führt [11]. Die Frage zu den Th1/Th17-Zellen erhielt auch neue Impulse durch eine rezente Publikation zur oft schwerer verlaufenden Down-Syndrom-assoziierten Arthritis, die eine Verminderung von Treg-Zellen und eine signifikante Vermehrung von polyreaktiven Th1/Th17-Zellen zeigte, die die Chemokinrezeptoren beider T-Zell-Subtypen exprimieren, CCR6 bzw. CXCR3 [12].

Interessanterweise könnte die Plastizität von Helfer-T-Zellen auch die unterschiedlichen Krankheitsverläufe sowie das zum Teil unterschiedliche Therapieansprechen bei der JIA erklären. Patienten mit einer milden, remittierenden Erkrankung (persistierende oligoartikuläre JIA) zeigen in der Synovialflüssigkeit eine signifikant höhere Anzahl von FoxP3-positiven CD4+-Helfer-T-Zellen, die hohe Expressionswerte von CD25 (IL-2Rezeptor) aufweisen, als Patienten mit einem schweren Krankheitsverlauf (extended oligoartikuläre JIA) und eine Verschiebung der Th17/Treg-Balance. Th17-Zel- len, die IL-17, IL-22 und IL-21 produzieren, sind bei der Extended Oligoarthritis deutlich vermehrt $[4,13]$. Zusätzlich erweisen sich die Treg-Zellen, die bei schweren Verlaufsformen gefunden werden, als dysfunktional insbesondere in Bezug auf ihre Suppressionsfähigkeit von proinflammatorischen Effektor-T-Zellen [14, 15]. Ähnliche Ergebnisse konnten auch für andere Autoimmunerkrankungen, wie z. B. die systemische Sklerose gezeigt werden [16, 17]. Auch hier war der Einfluss von epigenetischer Regulation insbesondere durch Methylierung am FoxP3-Promotor und Enhancer erkennbar, was zu einer schlechteren Stabilität des FoxP3+-Treg-Phänotyps und indirekt zu einer reduzierten Suppressionsfähigkeit der Treg-Zellen führt.

Interessant ist, dass Th17- und Treg-Zellen eine deutliche Verwandtschaft aufweisen. Hierbei können Treg-Zellen Merkmale von Th17-Zellen annehmen, wie RORC-Ko-Expression mit FoxP3, Hochexpression des CCR6-typischen Chemokinrezeptors und Produktion von IL-17 [18]. Diese Th17-ähnlichen Treg-Zellen sind möglicherweise ein induzierter Treg-Phänotyp (iTreg) aus naiven Th0-Zellen oder sind aus präexistenten Treg-Zellen unter dem Einfluss von IL-1 $\beta$ und IL-6, sowie hohen TGF- $\beta$-Dosen und IL-23, entstanden. Unklar ist, ob diese Th17/Treg-Zellen regulierende Funktionen ausüben können oder keine „echten“ Treg-Zellen mehr sind. Es wird vermutet, dass sich gerade dieser Th17/Treg-Zell-Subtyp wieder in Treg konvertieren kann, wenn das inflammatorische Milieu nachlässt. Dies wäre eine Chance für die Therapie mit hemmenden Antikörpern gegen die TNF- $\alpha$-, IL-1 $\beta$-, IL-6-, IL-23- und IL-17-Signalwege eine dauerhafte Rekonstitution der körpereigenen T-ZellRegulation zu erreichen.

Das Wiedererreichen einer T-Zell-Kontrolle durch suppressive Treg-Zellen konnte bereits für eine Inhibition von TNF- $\alpha$ und von IL-6 bei der rheumatoiden Arthritis gezeigt werden [19-23]. Etanercept, ein Anti-TNF- $\alpha$-Rezeptormolekül, führte zu einer Erhöhung der Th17- und Th17/Th1-transitorischen T-Zell-Subpopulation und reduzierte die CD161+-nicht-klassischen Th1-Zellen, ohne die Proportion der CD161-negativen klassischen Th1-Zellen zu beeinflussen [10]. Etanercept inhibierte den TNF- $\alpha$-getriebenen Shift von Th17 zu nicht klassischen Th1-Zellen [8]. Interessant ist der Einfluss von bereits zugelassenen oder in Entwicklung befindlichen Biologika, die IL-1 $\beta$ und IL-23, weitere Th17-induzierende Zytokine, hemmen oder von Janus-Kinase-(JAK)-Inhibitoren, die abhängig von ihrer Selektivität der verschiedenen JAK-1-, JAK-2- und JAK-3-Moleküle eine Vielzahl von Zytokine beeinflussen, die in der Plastizität von T-Helfer-Zellen beteiligt sind. Hierzu gilt es, Ergebnisse der Grundlagenforschung und der klinischen Studien abzuwarten. Auch Medikamente, die auf die Modifikation der Methylierung von Transkriptionsfaktoren abzielen und damit bestimmte T-Helfer-Zell-Subtypen stabilisieren oder destabilisieren könnten, sind in frühen Phasen der Entwicklung. Die zunehmenden Bestrebungen, Arthritis-spezifische Antigene zu identifizieren, rücken zumindest bei der rheumatoiden Arthritis bereits die Möglichkeit ins Licht, Antigen-spezifische Treg aus naiven CD4+-Th0Zellen oder sogar aus Effektor-T-Zellen generieren zu können. Dies könnte gelingen zusammen mit einer Induktion von FoxP3 und der Stabilisierung durch epigenetische Modifikation der Treg-Zell-Signatur-Gene [11, 24].

Zusammenfassend eröffnet die Erkenntnis zur Plastizität der T-Helfer-Zellen bei der JIA und anderen Formen der Autoimmun- 
arthritis ein neues Feld für das Verständnis zur Immunmodulation durch Biologika und Small molecules, wie JAK-Inhibitoren.

\section{Interessenkonflikt}

Die Autorin gibt folgende Beziehungen an: Vortragshonorare, Beratungshonorare und Forschungsförderung von AbbVie, Chugai Roche, Novartis, MSD, Janssen-Cilag, GSK, Pfizer, Baxter, SOBI

Literatur

[1] Lin YT, Wang CT, Gershwin ME et al. The pathogenesis of oligoarticular/polyarticular vs systemic juvenile idiopathic arthritis. Autoimmun Rev 2011; 10: 482-489

[2] Mahendra A, Misra R, Aggarwal A. Th1 and Th17 predominance in the enthesitis-related arthritis form of juvenile idiopathic arthritis. J Rheumatol 2009; 36: 1730-1706

[3] Sakaguchi S, Miyara M, Costantino CM et al. FOXP3+ regulatory T cells in the human immune system. Nat Rev Immunol 2010; 10: 490-500

[4] Nistala K, Wedderburn LR. Th17 and regulatory T cells: rebalancing pro- and anti-inflammatory forces in autoimmune arthritis. Rheumatology (Oxford) 2009; 48: 602-606

[5] Nistala K, Moncrieffe H, Newton KR et al. Interleukin-17-producing $T$ cells are enriched in the joints of children with arthritis, but have a reciprocal relationship to regulatory T cell numbers. Arthritis Rheum 2008; 58: 875-887

[6] Olivito B, Simonini G, Ciullini S et al. Th17 transcription factor RORC2 is inversely correlated with FOXP3 expressin in the joints of children with juvenile idiopathic arthritis. J Rheumatol 2009; 36: 2017-2024

[7] Maggi L, Cosmi L, Simonini G et al. T cell subpopulations in juvenile idiopathic arthritis and their modifications after biotherapies. Autoimmun Rev 2016; 15: 1141-1144

[8] Cosmi L, Cimaz R, Maggi L et al. Evidence of the transient nature of the Th17 phenotype of CD4+CD161+T cells in the synovial fluid of patients with patients with juvenile idiopathic arthritis. Arthritis Rheum 2011; 63: 2504-2515

[9] Nistala K, Adams S, Cambrook H et al. Th17 plasticity in human autoimmune arthritis is driven by the inflammatory environment. Proc Natl Acad Sci U S A 2010; 107: 14751-14756
[10] Maggi L, Cimaz R, Capone M et al. Brief report: etanercept inhibits the tumor necrosis factor $\alpha$-driven shift of Th17 lymphocytes toward a nonclassic Th1 phenotype in juvenile idiopathic arthritis. Arthritis Rheumatol 2014; 66: 1372-1377

[11] Mazzoni A, Santarlasci V, Maggi L et al. Demethylation of the RORC2 and IL17A in human CD4+T lymphocytes defines Th17 origin of nonclassic Th1 cells. J Immunol 2015; 194: 3116-3126

[12] Foley C, Floudas A, Canavan M et al. Increased T cell plasticity with dysregulation of $\mathrm{T}$ follicular helper, $\mathrm{T}$ peripheral helper and $\mathrm{T}$ regulatory cell responses in children with JIA and Down syndrome-associated arthritis. Arthr Rheumatol 2019. https://doi.org/10.1002/art.41150

[13] Annunziato F, Cosmi L, Liotta F et al. Main features of human T helper 17 cells. Ann N Y Acad Sci 2013; 1284: 66-70

[14] Wehrens EJ, Mijnheer G, Duurland CL et al. Functional human regulatory $T$ cells fail to control autoimmune inflammation due to $\mathrm{PKB} / \mathrm{c}$-akt hyperactivation in effector cells. Blood 2011; 118: 3538-3548

[15] Haufe S, Haug M, Schepp C et al. Impaired suppression of synovial fluid CD4+CD25-T cells from patients with juvenile idiopathic arthritis by CD4+CD25+ Treg cells. Arthritis Rheum 2011; 63: 3153-3162

[16] Almanzar G, Klein M, Schmalzing $M$ et al. Disease Manifestation and Inflammatory Activity as Modulators of Th17/Treg Balance and RORC/ FoxP3 Methylation in Systemic Sclerosis. Int Arch Allergy Immunol 2016; 171: 141-154

[17] Ugur E, Simon D, Almanzar G et al. Increased proportions of functionally impaired regulatory $T$ cell subsets in systemic sclerosis. Clin Immunol 2017; 184: 54-62

[18] Kleinewietfield M, Hafler DA. Plasticity of human Treg and Th17 cells. Sem Immunol 2013; 25: 305-312

[19] Ehrenstein MR, Evans JG, Singh A et al. Compromised function of regulatory $T$ cells in rheumatoid arthritis and reversal by anti-TNFalpha therapy. J Exp Med 2004; 200: 277-285

[20] McGovern JL, Nguyen DX, Notley CA et al. Th 17 cells are restrained by Treg cells via the inhibition of interleukin-6 in patients with rheumatoid arthritis responding to anti-tumor necrosis factor antibody therapy. Arthritis Rheum 2012; 64: 3129-138

[21] Nadkarni S, Mauri C, Ehrenstein MR. Anti-TNF-alpha therapy induces a distinct regulatory $T$ cell population in patients with rheumatoid arthritis via TGF-beta. J Exp Med 2007; 204: 33-39

[22] Bystrom J, Clanchy Fl, Taher TE et al. TNFa in the regulation of Treg and Th17 cells in rheumatoid arthritis and other autoimmune inflammatory diseases. Cytokine 2018; 101: 4-13

[23] Kim EY, Moudgil KD. Immunomodulation of autoimmune arthritis by pro-inflammatory cytokines. Cytokine 2017; 98: 87-96

[24] Komatsu N, Takayanagi H. Regulatory T cells in arthritis. Prog Mol Biol Transl Sci 2015; 136: 207-215 